\title{
Microscopic and chemical analysis of room temperature UV laser annealing of solution- based zinc-tin-oxide thin films
}

\author{
Ji-Hwan Kwon', Seunghoon Jang ${ }^{2}$, Hyuk Jin Kim³, Beom Soo Joo ${ }^{3}$, Kwang Nam Yư ${ }^{3}$, Eunjip Choi ${ }^{3}$, Moonsup Han ${ }^{3}$,
} Jong Hyuk Park ${ }^{4}$ and Young Jun Chang ${ }^{3^{*}}$ (D)

\begin{abstract}
As a promising transparent semiconducting oxide (TSO) candidate, zinc-tin-oxide (ZTO) thin films were fabricated by combining solution coating and ultraviolet (UV) laser annealing. Instead of external heating, an intense UV laser was applied to transform sol-gel coatings via surface heating and photoexcited dissociation into oxide films. The laser-induced phase transformation was extensively investigated with synchrotron-based X-ray photoelectron spectroscopy (XPS), transmission electron microscopy (TEM), and electron energy loss spectroscopy (EELS). The chemical states and microscopic distributions of oxygen, zinc, and tin were significantly modified during the laser irradiation. Relative oxidation and surface migration between zinc and tin gradually evolved toward a combination of $\mathrm{ZnO}_{1-x}$ host and $\mathrm{SnO}_{2-y}$ grains. Our results present deeper insight into the use of UV laser annealing for developing a room temperature (RT) fabrication method of TSO thin films and other relevant solution coatings.
\end{abstract}

Keywords: $\mathrm{ZnSnO}_{x}$ Ultraviolet laser annealing, Sol-gel, XPS, TEM

\section{Introduction}

Transparent flexible electronics highly demand compatible new materials, such as transparent conductors and semiconductors. Indium-tin oxide (ITO) is the most widely used for transparent conducting oxides (TCOs) or semiconducting oxides (TSOs) (Minami, 2008; Noh et al., 2016; Hong et al., 2017). However, the high cost of indium has led to extensive efforts to find alternative materials, such as zinc oxide ( $\mathrm{ZnO}$ ) (Banerjee, 2006; Kim et al., 2012), $\mathrm{BaSnO}_{3}$ (Seo et al., 2014), or graphene (Bae et al., 2010; Kang et al., 2016; Choi et al., 2015). Metal oxide candidates require a new kind of low-temperature oxidation process for application to the thermally unstable flexible substrates. Therefore, low-temperature oxidation for solution-based oxide thin film fabrication is one of the highly demanded process technologies for

\footnotetext{
* Correspondence: yjchang@uos.ac.kr

${ }^{3}$ Department of Physics, University of Seoul, Seoul 02504, Republic of Korea Full list of author information is available at the end of the article
}

flexible electronic device applications (Banerjee, 2006; Kim et al., 2012; Fernandes et al., 2018).

Among many $\mathrm{TCO} / \mathrm{TSO}$ candidates, $\mathrm{ZnO}$ is one of the most studied materials, along with its other applications in photocatalysis (Kang et al., 2016; Lee et al., 2017) and photovoltaics (Izaki et al., 2007), due to its semiconducting properties, such as direct bandgap and photoluminescence (Oh et al., 2018). All these applications rely on the band structure and defect properties in the solution-derived $\mathrm{ZnO}$ thin films, which are typically fabricated at low temperature. In addition, chemical doping, such as $\mathrm{Sn}$ or In, gives rise to the carrier injection and electrical conductivity even in the amorphous phase. Local distribution of dopant in the film geometry further influences the interfacial electrical contact properties or electrical mobility due to charge scattering (Sandu et al., 2003). Thus, it is important to investigate the electronic structure, chemical distribution, and defect characteristics of zinc-tin-oxide (ZTO) during lowtemperature synthesis (Lim et al., 2012). 
Sol-gel film synthesis requires high-temperature annealing above $400{ }^{\circ} \mathrm{C}$ for both chemical bond dissolution and densification. Different approaches have been taken to reduce the annealing temperature, including ultraviolet (UV) lamp, or UV laser irradiation. UV light has enough energy to stimulate the chemical bonds to break apart so that UV lamp annealing reduces the annealing temperature down to $150{ }^{\circ} \mathrm{C}$. Unfortunately, UV lamp annealing is only applicable to a few kinds of solution, of which optical absorption is significant at the given UV wavelength. Overcoming this limitation requires more intense UV light, such as UV laser. The intense laser light can stimulate the solution coating with even small absorption coefficient due to multiple light scattering. The absorbed laser light is then transformed into heat inside the coated layer without damaging the underlying substrates.

During the UV laser annealing, the short pulses (20 ns) of the excimer laser rapidly dissociate the molecular bondings followed by surface heating. Such rapid chemical and physical transformation requires precise chemical and physical analysis. Previous works have identified the chemical dissociation and inorganic bond formation by using X-ray photoemission spectroscopy (XPS) (Kim et al., 2012; Kim et al., 2019). Unfortunately, highresolution chemical and microstructure analyses have not been performed for precisely monitoring the chemical states of oxygen and cations. Especially, core levels of $\mathrm{Zn}$ and Sn change very little in energy (0.8 and 0.65 $\mathrm{eV}$, respectively) during oxidation. Comprehensive understanding of the laser-induced process necessitates research into such chemical and morphology changes for both the surface and interior regions.

Herein, we report the chemical and morphological changes of the ZTO thin films during the UV laser annealing. We prepared the ZTO thin films via UV laser irradiation, varying the number of laser pulses at room temperature (RT). We investigated the bonding and microstructural structural properties of the surface and the interior region of the ZTO thin film by using highresolution XPS measurements and transmission electron microscopy (TEM), along with electron energy loss spectroscopy (EELS) and energy dispersive X-ray spectroscopy (EDS). The UV laser pulses modified the bonding characteristic of zinc, tin, and oxygen, effectively converting the sol-gel coating to oxide films for a short period of time without external heating.

\section{Methods/experimental}

We prepared sol-gel films on $\mathrm{SiO}_{2} / \mathrm{Si}$ wafer by the following steps. We spin coated the ZTO solution, in which $\mathrm{ZnCl}_{2}$ and $\mathrm{SnCl}_{2}$ salts are dissolved in 2-ME solvent, with $3000 \mathrm{rpm}$ for $1 \mathrm{~min}$ to fabricate coated films with a uniform thickness of $\sim 100 \mathrm{~nm}$. Then, we applied the UV laser pulse using an excimer laser (COMPEXPro $201, \mathrm{KrF}, \lambda=248 \mathrm{~nm}$ ) on the samples kept in a vacuum chamber with a controlled oxygen pressure of 100 mTorr. During the UV laser irradiation, we only changed the number of pulses, but the power of the UV laser and the repetition rate were fixed at $20 \mathrm{~Hz}$ and $0.8 \mathrm{~W}$, respectively. No external heating was applied. The overall procedure of fabrication of the ZTO films using the excimer laser is illustrated in Fig. 1a.

We analyzed the physical properties of the films using optical spectroscopy, synchrotron-based XPS, and TEM. To study the optical properties, we performed spectroscopic ellipsometry (V-VASE (J, A. Wollam Co., Inc.)) in the range of $0.74-5.5 \mathrm{eV}$ at RT. To examine the changes of the chemical bonding and the stoichiometry, we performed XPS measurements at beamline 4A1 of Pohang Light Source. In the XPS experiment, we introduced the samples into an ultra-high vacuum analysis chamber, with a base pressure of under $5 \times 10^{-10}$ Torr, without any prior surface treatments. The energy resolution was $100 \mathrm{meV}$ for the synchrotron XPS data. The position of the Fermi level $\left(\mathrm{E}_{\mathrm{F}}\right)$ was calibrated by measuring the photoemission spectra of the sputter-cleaned polycrystalline gold surface, which was in electrical contact with the sample. For TEM measurements, the cross-sectional TEM samples were prepared by mechanical polishing and ion beam milling. The ion beam milling was done using a Gatan model 691 precision Ion Polishing System. To minimize ion beam damages from the milling, a fine milling at $2.0 \mathrm{kV}$ was performed after typical ion milling operation. The conventional high-resolution TEM experiment was performed using JEOL-3000F operated at $300 \mathrm{kV}$, and the scanning transmission electron microscopy (STEM) and EELS/EDS were performed using JEOL-2200FS operated at $200 \mathrm{kV}$. The inner cutoff angle for HAADF detector was 100 mrad for STEM imaging.

\section{Results \\ Optical property}

To investigate the electronic structure change during the UV laser annealing process, we analyzed the optical absorption spectra, as shown in Fig. 1b. We plotted the Tauc plot with the squared optical absorption coefficient multiplied by the photon energy under the assumption of being an indirect bandgap semiconductor, i.e., indirect bandgap in $\mathrm{SnO}_{2}$ and direct bandgap in $\mathrm{ZnO}$ (Lim et al., 2012; Mishra et al., 1995). The as-coated ZTO sol-gel film and the $1 \mathrm{p}$ sample did not show an inter-band absorption edge near $4 \mathrm{eV}$, whereas the $30 \mathrm{p}$ and $20,000 \mathrm{p}$ samples showed bandgap absorption edges at 4.0 and $3.9 \mathrm{eV}$, respectively. These observations indicate that only a few laser pulses ( 30 pulses) were needed to convert the sol-gel film into crystallized bonding states with bandgap formation. Between the 30p and 20,000p 




(b)
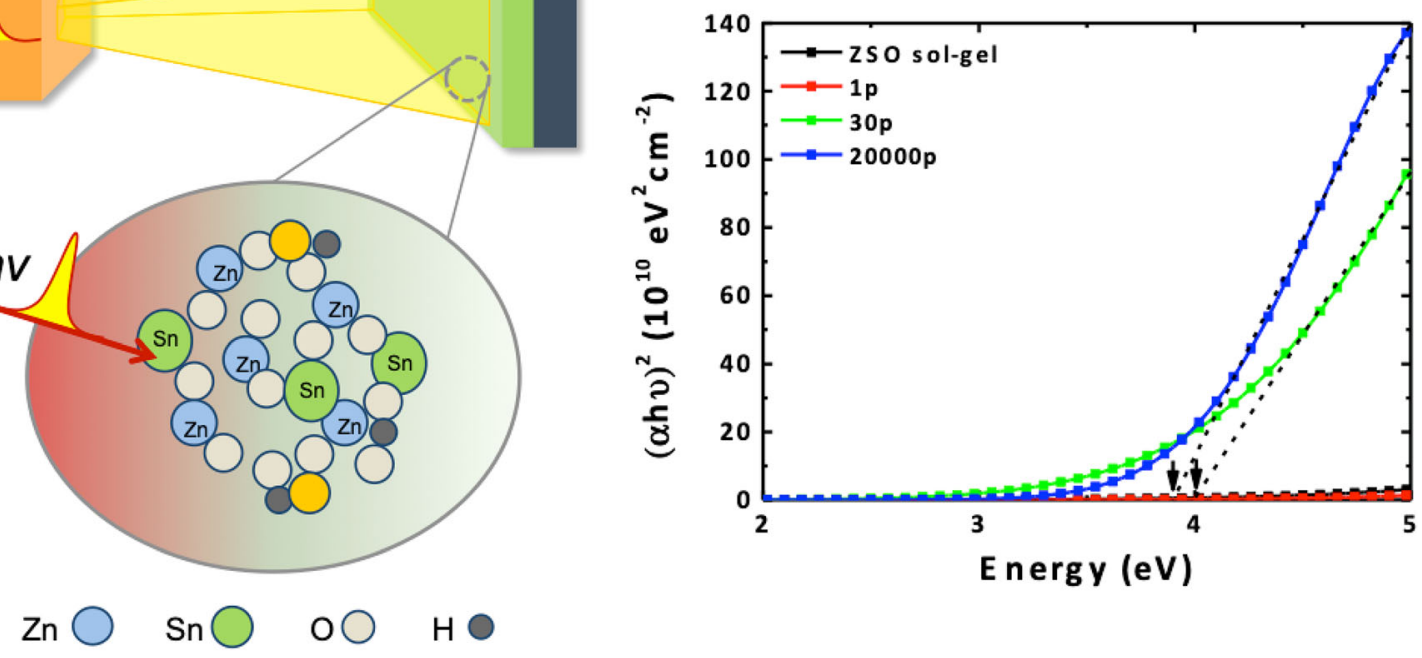

Fig. 1 a Schematic figure of excimer laser annealing of sol-gel thin films at room temperature. $\mathbf{b}$ Optical absorption spectra (a) of the ZTO $\left(\mathrm{ZnSnO}_{x}\right)$ films with variation of laser annealing duration. The red lines represent the linear extrapolation of (ahv) $)^{2}$ to the $x$-axis, for estimating the bandgap values

samples, the extended laser annealing may have increased the fraction of laser annealed crystalline phases, which is discussed below in detail.

\section{Chemical state changes of oxygen, zinc, and tin}

Figure 2 shows the relative composition of the ZTO films measured by XPS over the number of excimer laser pulses. The relative stoichiometry changes of the ZTO films were estimated by normalizing the integrated intensities of the $\mathrm{Zn} 3 d, \mathrm{Sn} 3 d, \mathrm{O} 2 p$, and $\mathrm{Cl} 2 p$ peaks with atomic sensitivity factors. In addition to the typical XPS measurement geometry with photoelectron emission angle of $0^{\circ}$, i.e., surface normal direction, the photoelectron emission with $60^{\circ}$ to surface normal was used to obtain more surface sensitive signals. Since this lowangle emission contains more surface sensitive signals compared to the typical emission angle, we refer to the emission angle of $0^{\circ}$ (surface normal) as the "near-surface" spectrum and to the emission angle of $60^{\circ}$ as the "top-surface" spectrum. Comparing these two emission
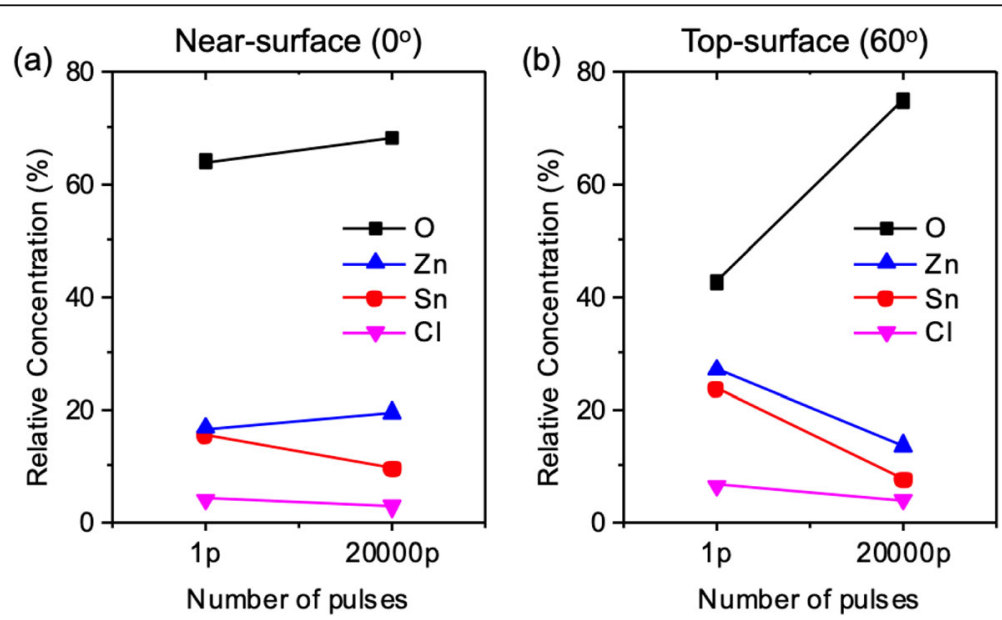

Fig. 2 Relative composition of ZTO films for a the near-surface region (photoelectron emission angle of $0^{\circ}$ ) and $\mathbf{b}$ the top-surface region $\left(60^{\circ}\right)$. As the number of laser pulse increases, oxygen content increases and chlorine content decreases for both measurement geometries. 
angles enabled us to compare the chemical information between the near-surface and top-surface regions.

The composition ratio of the ZTO film was significantly changed after the long (20,000 pulses) UV laser annealing. The oxygen content showed the most significant increase in both photoelectron emission angles. Comparably, the top-surface region showed a more drastic increase of oxygen content from 43 to $75 \%$, as shown in Fig. $2 \mathrm{~b}$, implying that the UV laser annealing process effectively dissociates the molecular bonding with the chlorine and promotes the oxidation of metals from the top-surface region. Due to the increase of oxygen, the relative concentrations of $\mathrm{Zn}$ and $\mathrm{Sn}$ were decreased, as expected, at the topsurface, as shown in Fig. 2b ( $\mathrm{Sn} 24 \% \rightarrow 8 \%$ and $\mathrm{Zn}$ $27 \% \rightarrow 14 \%)$. In contrast, at the near-surface region in Fig. 2a, the $\mathrm{Zn}$ concentration slightly increased ( $\mathrm{Zn}$ $17 \% \rightarrow 20 \%)$, whereas the Sn concentration decreased (Sn 15\% $\rightarrow$ 10\%) after long UV laser annealing. The $\mathrm{Zn} / \mathrm{Sn}$ ratio increased both at the near-surface and top-surface regions, which is attributed to the significant decrease of Sn concentration. Overall, the ZTO films showed dramatic variation of surface stoichiometry after the pulsed laser irradiation.

We investigated the chemical state of each element in the ZTO film via detailed analysis of XPS spectra (Wencka et al., 2018). Figure 3 shows the XPS spectra (emission angle of $0^{\circ}$ ) of $\mathrm{O} 1 s, \mathrm{Zn} 3 d$, and $\mathrm{Sn} 3 d$, which changed over the number of pulsed laser irradiation.
The $\mathrm{O} 1 s$ core-level spectrum contained three different forms of oxygen: $\mathrm{M}-\mathrm{O}_{\mathrm{v}}(531.4 \mathrm{eV}), \mathrm{M}-\mathrm{O}(530.2 \mathrm{eV})$, and $\mathrm{M}-\mathrm{OH}(532.7 \mathrm{eV})$. The peak labeled $\mathrm{M}-\mathrm{O}_{\mathrm{v}}$ was assigned to the bonding between metal and the oxygen vacancy, whereas the peaks $\mathrm{M}-\mathrm{O}$ and $\mathrm{M}-\mathrm{OH}$ originated from the metal-oxygen and metal-hydroxy bonding, respectively (Kim et al., 2012; Kwoka et al., 2005). In the case of $\mathrm{Zn}$ and $\mathrm{Sn}$, the $\mathrm{Zn} 3 d$ and Sn $3 d$ spectra were deconvoluted into two peaks by peak fitting. For $\mathrm{Zn}$, the metal $\mathrm{Zn}$ and $\mathrm{ZnO}$ phases were found for the $\mathrm{Zn} 3 d$ peak, whereas two forms of oxide were observed for the Sn $3 d$ peak: $\mathrm{SnO}$ and $\mathrm{SnO}_{2}$ phase. Interestingly, the metal $\mathrm{Sn}$ phase was not observed in the XPS result; however, cross-sectional TEM and EELS results revealed the metal Sn phase in the form of a nanocrystalline phase that is distributed inhomogeneously near the bottom of the ZTO film, which is discussed below.

After peak fitting, we analyzed the changes of chemical state quantitatively for both near-surface and top-surface regions, as displayed in Fig. 4. As the number of laser pulse was increased, the amounts of $\mathrm{M}-\mathrm{OH}$ and $\mathrm{M}-\mathrm{O}$ bondings decreased, whereas that of $\mathrm{M}-\mathrm{O}_{\mathrm{v}}$ bonding increased. The decrease of $\mathrm{M}-\mathrm{OH}$ bonding is attributed to the decrease of moisture and residue in the ZTO film, which is due to the heating and dissolving effect of UV. The $\mathrm{M}-\mathrm{O}$ bonding decreased despite the increase in the oxygen content after laser irradiation on the ZTO film, suggesting that the $\mathrm{ZnO}$ and $\mathrm{SnO}$ formed by laser irradiation
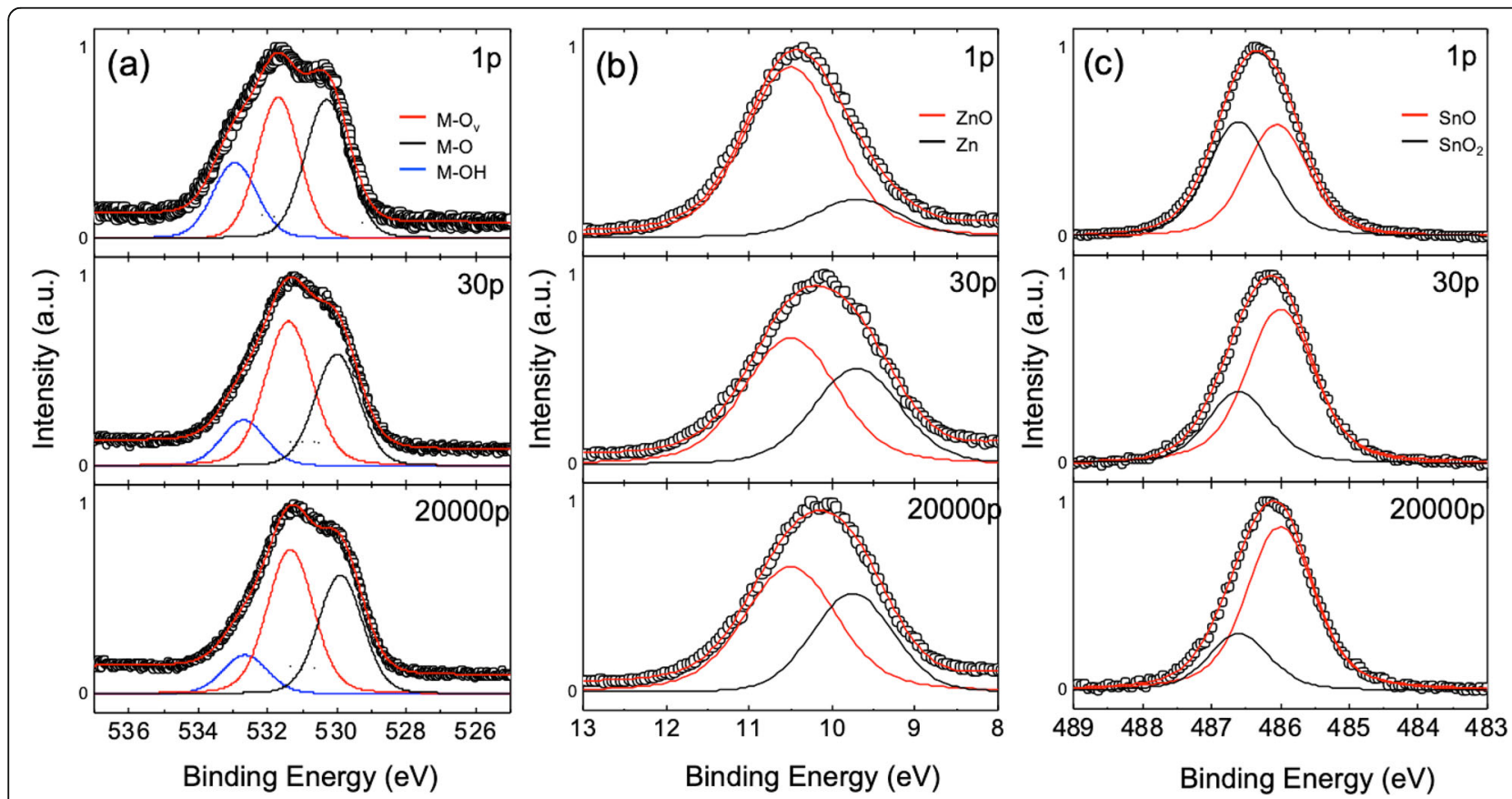

Fig. 3 The changes of XPS core-level spectra of $\mathbf{a} O 1$ s, $\mathbf{b}$ Zn 3d, and $\mathbf{c}$ Sn 3d in ZTO thin films over the number of laser pulses (photoelectron emission angle of $0^{\circ}$ ) 
(a)

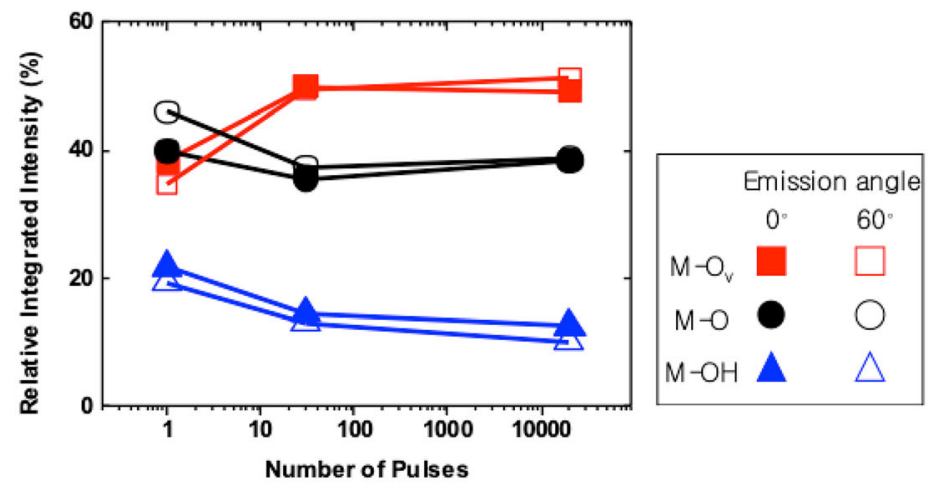

(b)

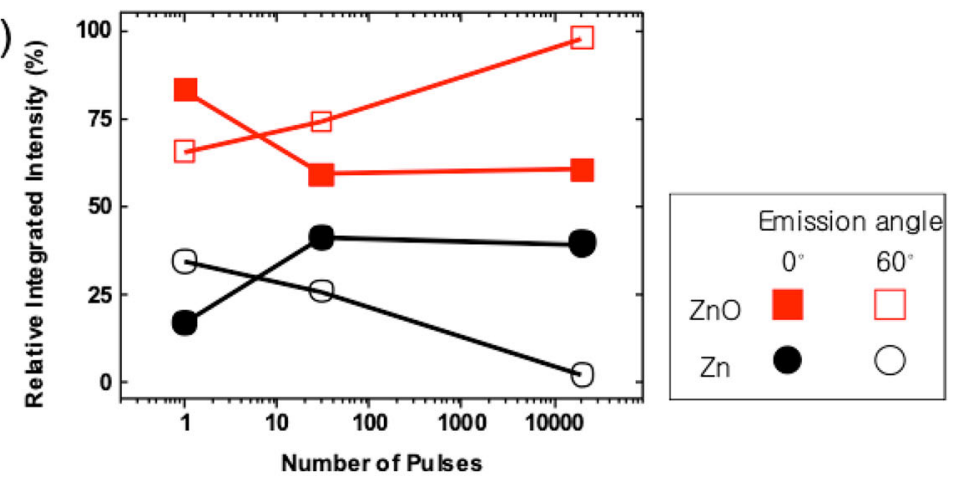

(c)

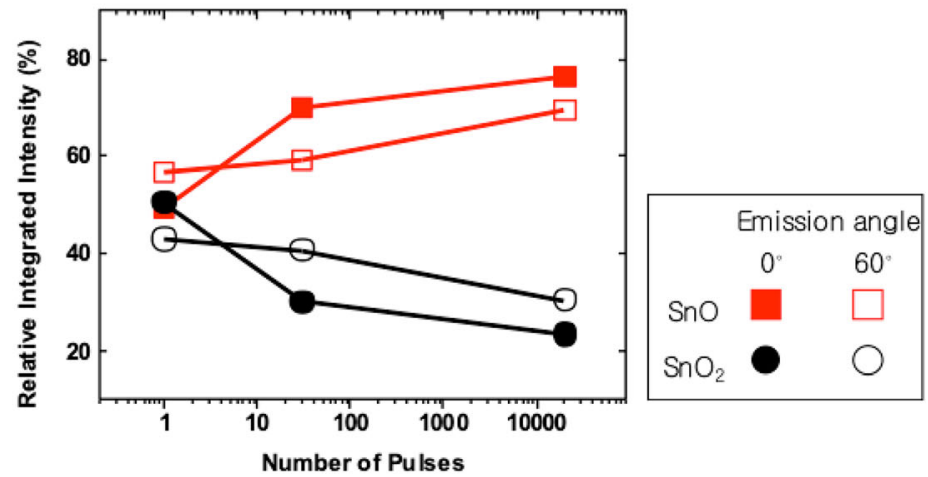

Fig. 4 The changes of chemical state of $\mathbf{a} \mathrm{O}, \mathbf{b} \mathrm{Zn}$, and $\mathbf{c} \mathrm{Sn}$ in ZTO as a function of the number of laser pulses

mostly contained $\mathrm{M}-\mathrm{O}_{\mathrm{v}}$ bonding in their oxide form. In case of $\mathrm{Zn}$, the relative concentration of $\mathrm{ZnO}$ decreased from 83 to 59\%, while that of $\mathrm{Zn}$ metal increased from 17 to $41 \%$ in the near-surface region after 30 pulses. Further irradiation caused no noticeable changes, resulting in a final composition of $\mathrm{ZnO}$ : $\mathrm{Zn}=61: 39$. In the top-surface region, however, the concentration of $\mathrm{ZnO}$ continued increasing during UV laser annealing, so the mostly $\mathrm{ZnO}$ phase was formed on the top surface $(\mathrm{ZnO}: \mathrm{Zn}=98: 2)$. We note that the intensity difference of $\mathrm{ZnO}$ and $\mathrm{Zn}$ between the top surface and the near surface is significantly larger than that of $\mathrm{O}$ and $\mathrm{Sn}$ in Fig. 4. We speculate that $\mathrm{Zn}$ at the top surface can easily change its oxidation state sensitively to the exposed atmosphere during the given temperature rise. Contrast of $\mathrm{ZnO}$ ratios between the top surface and near-surface regions can be understood with lack of enough oxygen supply in the deeper region, where metal-oxygen bonding is also reduced for $\mathrm{SnO}$ and $\mathrm{M}-\mathrm{O}_{\mathrm{v}}$ components. We also note that such enhanced oxidation at the top surface provides significant implication for understanding the electrical properties at the oxidemetal junctions, such as complex heterostructures in display devices.

Finally, Sn showed very different behavior from $\mathrm{Zn}$. After long UV laser annealing, the relative intensity of $\mathrm{SnO}$ increased from 49 to $77 \%$, while that of $\mathrm{SnO}_{2}$ decreased from 51 to $23 \%$ in the near surface. In the topsurface region, $\mathrm{Sn}$ showed similar behavior: more $\mathrm{SnO}$ phase was formed than $\mathrm{SnO}_{2}$ phase after long UV laser annealing. The decrease of $\mathrm{SnO}_{2}$ concentration over the 
laser irradiation is attributed to the reduction environment for $\mathrm{SnO}_{2}$, which is caused by the laser heating effect at the surface (for example, surface temperature range of $600 \mathrm{~K} \sim 1700 \mathrm{~K}$ for laser density of $0.1 \sim 0.5 \mathrm{~J} / \mathrm{cm}^{2}$, respectively) (Grigoropoulos et al., 1996; Lee et al., 2016a; Nakajima et al., 2010). Indeed, it has been shown that $\mathrm{SnO}_{2}$ can be reduced into $\mathrm{SnO}$ at high temperature, which is part of the well-known thermochemical cycle reaction for producing hydrogen fuel for solar energy applications (Charvin et al., 2008). We believe that the reduced $\mathrm{SnO}$ could then be transformed into metallic $\mathrm{Sn}$, leaving nanocrystalline metallic Sn phases found in the bottom of the ZTO film, which is shown in the TEM images below.

\section{Microscopic morphology change and chemical redistribution}

In order to observe the overall morphology of the ZTO film, TEM was performed. The ZTO sample irradiated by the excimer laser with 20,000 pulses was chosen for the TEM observation. Figure 5 a shows a low magnification cross sectional TEM image of the ZTO thin films on $\mathrm{SiO}_{2} / \mathrm{Si}$ substrate. The $\mathrm{ZTO}$ film was grown with a uniform thickness of $\sim 40 \mathrm{~nm}$ on the $\mathrm{SiO}_{2} / \mathrm{Si}$ substrate. However, a higher magnification image revealed that the contrast of the ZTO film was not uniform: the contrast appears brighter near the surface of the ZTO film, whereas the bottom of the ZTO film shows a relatively dark contrast, as displayed in Fig. 5b. In addition, some secondary phases appearing as dark particles are observed near the film/substrate interface region, as indicated by the arrows in Fig. 5b. The secondary phases appear as oval shapes, a few tens of nanometer wide and $\sim 10 \mathrm{~nm}$ high, and are distributed irregularly near the film/substrate interface. The high magnification image in Fig. $5 \mathrm{c}$ shows nanocrystalline phases in the dark region of the ZTO film.

Figure 6a shows a STEM image of the ZTO film. The STEM image shows some dark spots with a diameter of $\sim 4 \mathrm{~nm}$, which are mostly found near the film surface. The dark spots extend into the bottom of the ZTO film in some areas. The contrast of the ZTO films appears not uniform, as shown in the TEM image (see Fig. 5b), which is attributed to the inhomogeneous distributions of $\mathrm{ZnO}_{\mathrm{x}}$ and $\mathrm{SnO}_{\mathrm{x}}$ with different densities due to nanoscale aggregation during the pulsed laser irradiation. On the other hand, the bright contrast region was observed in some areas of the bottom of the ZTO film. Since the contrast of the STEM image was proportional to $\sim \mathrm{Z}^{2}$ (Pennycook, 2002; Pennycook et al., 2018), this bright contrast region in the STEM image is ascribed to the higher atomic number of $\mathrm{Sn}$, i.e., Sn-rich phase (atomic numbers of $\mathrm{Sn}$ and $\mathrm{Zn}$ are 50 and 30, respectively). This
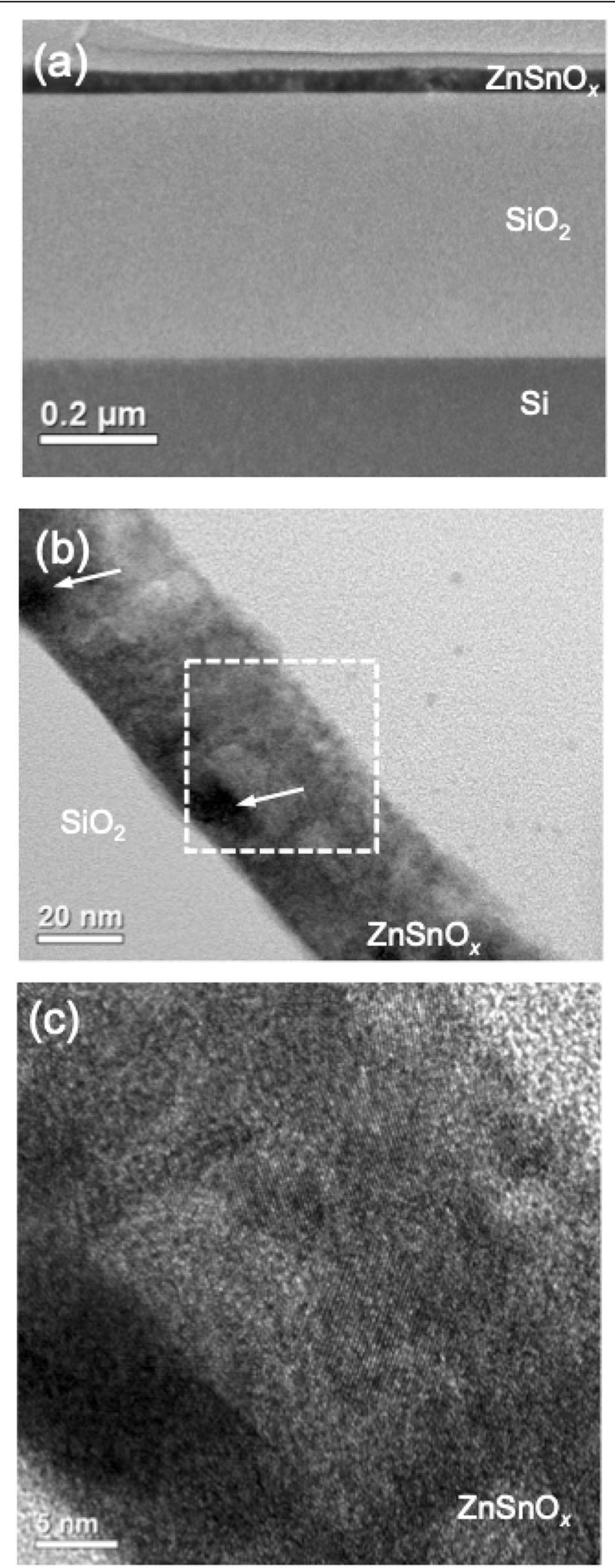

Fig. 5 a Low magnification TEM image of ZTO thin films on $\mathrm{SiO}_{2} / \mathrm{Si}$ substrate. The film is grown in uniform thickness of $40 \mathrm{~nm}$. $\mathbf{b}$ TEM image of ZTO film. Some dark contrast regions are observed near the film/substrate interface. c High magnification image of ZTO film, showing some nanocrystalline phases 

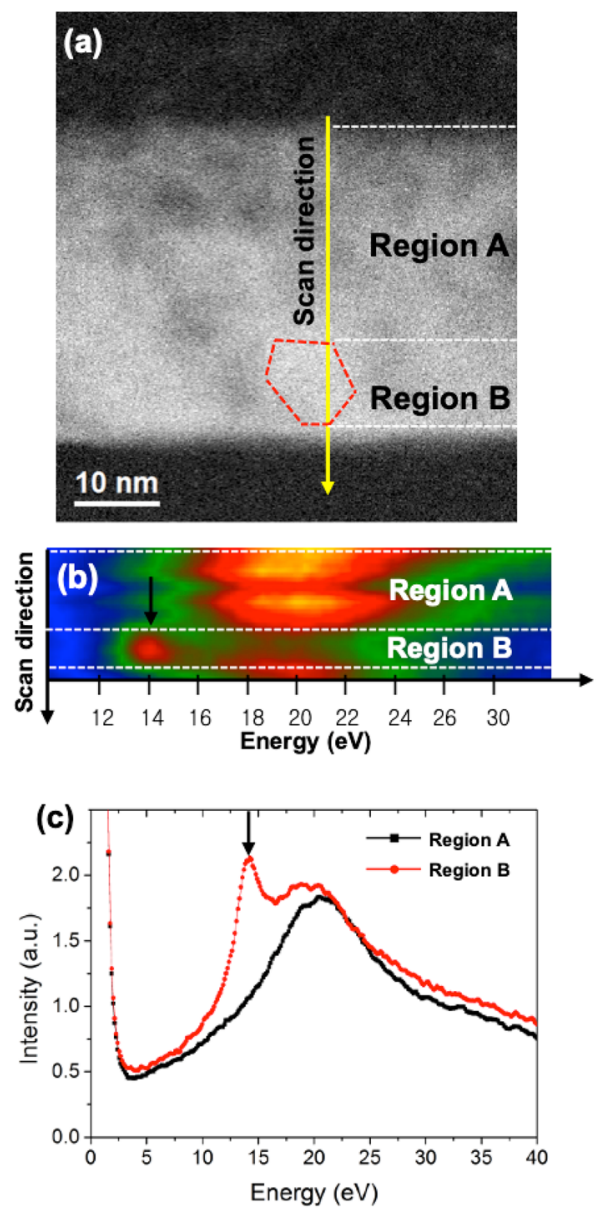

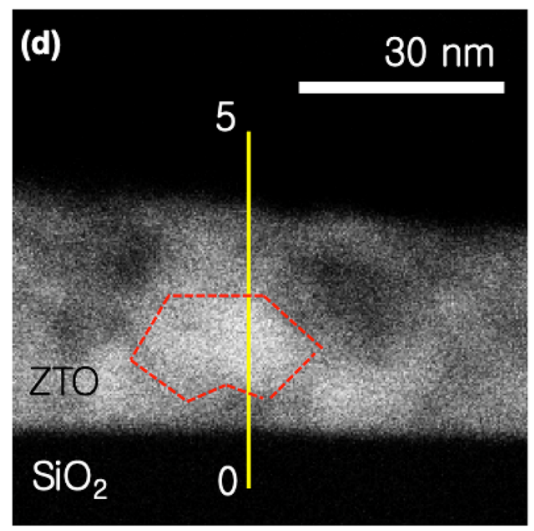

(e)

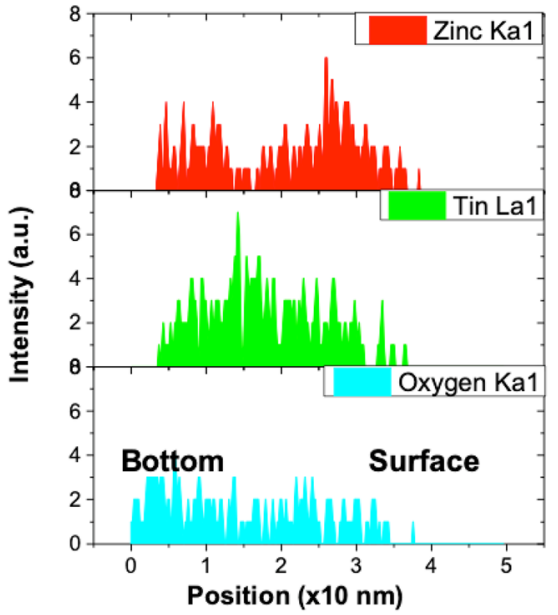

Fig. 6 a STEM image of the ZTO thin films on $\mathrm{SiO}_{2} / \mathrm{Si}$ substrate after excimer laser irradiation. $\mathbf{b}$ Low-loss EEL spectrum image across the ZTO thin film along the line scan in $\mathbf{a}$. The ZTO film is composed of two distinctive regions: upper (region A) and lower (region B) region. The region B contains additional plasmon peak at $\sim 14 \mathrm{eV}$. c Low-loss spectrum from region A and region B, respectively. d STEM image of ZTO thin films where EDS line scan was performed. e EDS results: Zn, Sn, and oxygen profiles along the line scan drawn in $\mathbf{d}$

bright contrast region in STEM image was consistent with the secondary phases observed in TEM mode.

In order to identify the secondary phases, low-loss EELS experiments were performed across the ZTO film containing the bright contrast region in the bottom, as shown in Fig. 6a. Figure 6 b shows a low-loss EEL spectral map of the ZTO film. The low-loss EEL spectral map revealed the ZTO film to be composed of two distinctive regions: an upper region (region $\mathrm{A}$ ) with a plasmon peak at $21 \mathrm{eV}$ and a lower region (region $\mathrm{B}$ ) with a peak at $\sim 14 \mathrm{eV}$ in addition to the peak at $21 \mathrm{eV}$. Although the upper region (region A) exhibited a contrast variation in the STEM image, no discernible change was found in the low-loss spectrum within the upper region, suggesting that the contrast variation in the STEM image was caused by the different film density rather than the different phases. The plasmon peak at $\sim 14 \mathrm{eV}$ is consistent with metallic Sn or Zn (Seiler et al., 1991; Widder et al., 1997), supporting the presence of metallic nanosized grains at the bottom. Furthermore, in Fig. $6 \mathrm{~d}$ and e, the EDS measurements showed that the Sn signal was distributed more in the bottom while the $\mathrm{Zn}$ signal dominated near the surface region. To address more about the complicated polycrystalline phases in the ZTO film, we performed structural analyses using highresolution TEM image and its fast Fourier transform (FFT). In Fig. 7, we found that the ZTO film is composed of many different phases, such as metals ( $\mathrm{Zn}$ and $\mathrm{Sn})$ and oxides $\left(\mathrm{ZnO}, \mathrm{SnO}, \mathrm{SnO}_{2}, \mathrm{ZnSnO}_{3}\right.$, and $\mathrm{Zn}_{2} \mathrm{SnO}_{4}$ ). Each of the measured $d$-spacings in the regions $\mathrm{A}$ and $\mathrm{B}$ of Fig. 7a could be found in one of the candidate phases, which confirms that the regions $\mathrm{A}$ and $B$ contain many different phases. Based on the EELS, EDS, and structural analysis results, we concluded that the secondary phases observed in the STEM image were metallic Sn phase located in the bottom of the ZTO films, while supporting a higher $\mathrm{Zn}$ concentration in the surface region, as observed in the XPS analysis. 


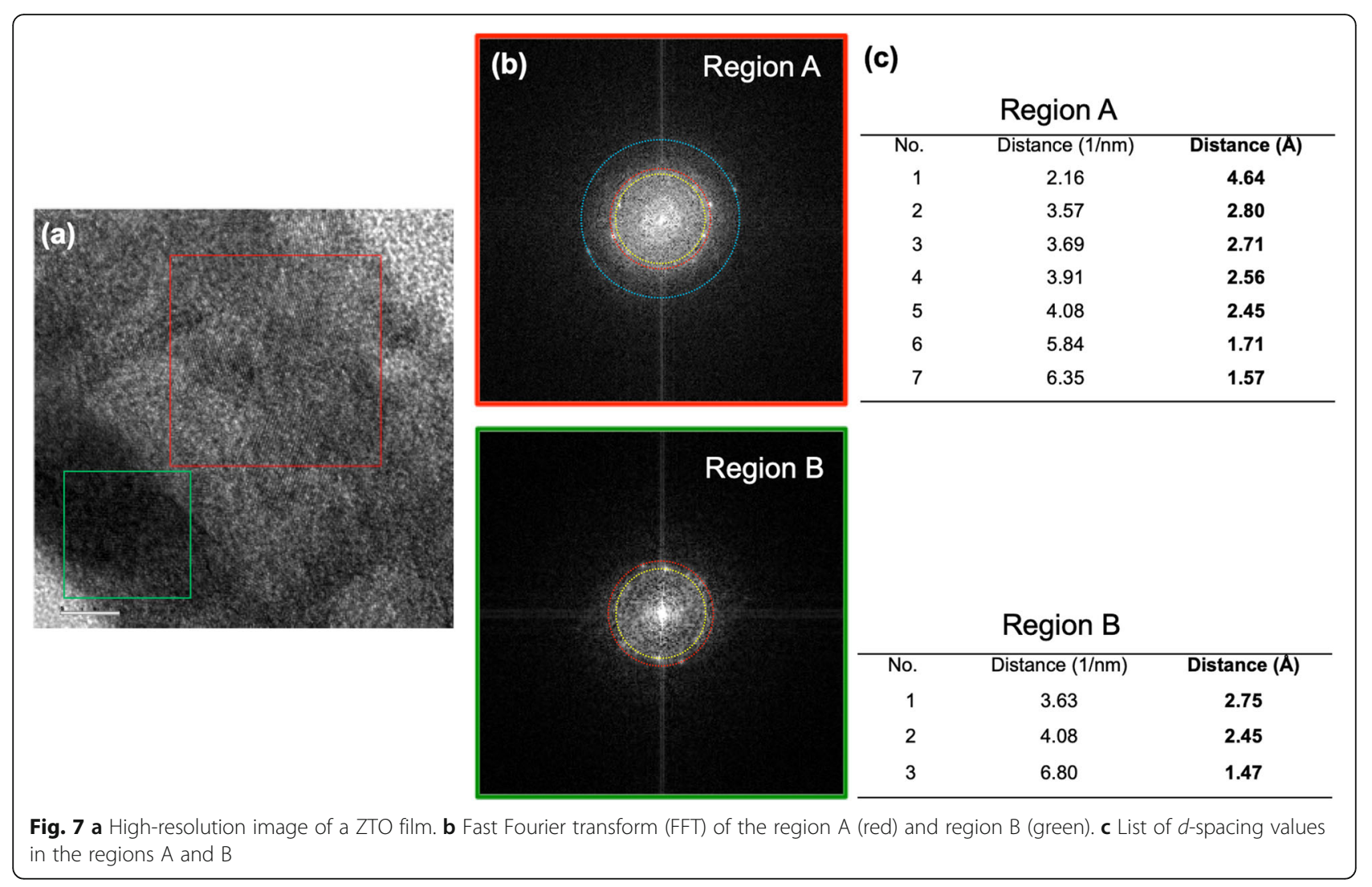

\section{Discussion}

We extensively analyzed TSO films during the RT UV laser annealing process, which effectively and quickly transformed sol-gel coatings into oxide films. Extensive XPS analysis revealed not only surface depletion of Sn, but also oxygen-reduced phase formation at the top surface, and thus provided important information on this electrical conducting surface layer for developing heterostructure devices with similar characteristics of TSO materials (Kim et al., 2012; Kim et al., 2019; Chang, 2018; Lutz et al., 2018). As complementary approaches, detailed cross-sectional TEM analysis provided microscopic evidence of a gradient density distribution from the surface to the bottom of the films. The STEM and EELS/EDS analyses clearly showed chemical migration to form $\mathrm{Zn}$-rich surface and Sn-rich bottom regions, which was consistent with the XPS results. Based on the observation of inhomogeneous distribution of $\mathrm{Zn}$ and $\mathrm{Sn}$ in the films, we can relate the compositional difference to temperature profile during the UV laser annealing. The top surface temperature rises up much higher than the deeper region, and such thermal gradient may redistribute more $\mathrm{Zn}$ near surface regions and more Sn toward bottom regions. In addition, such Zn-rich phases at the top surface should play an important role for determining the interfacial electrical contact properties, such as contact resistance or work function (Sandu et al., 2003). Hence, our extensive XPS and TEM analyses demonstrated both chemical and microscopic understanding of the UV laser annealing process and provided valuable insights for understanding the resulting electrical performance, which are applicable to the relevant fabrication processes on such TSO films (Hasan et al., 2012; Lee \& Cho, 2016b).

\section{Conclusions}

In summary, we utilized UV laser pulses to anneal a sol-gel ZTO coating into transparent oxide films at RT. After UV pulsed laser irradiation, ZTO thin films were successfully fabricated. The synchrotron-based XPS and detailed TEM measurements showed that the chemical state of oxygen, $\mathrm{Zn}$, and $\mathrm{Sn}$ was significantly changed, along with their redistribution, after laser irradiation. Our results demonstrated that RT UV laser annealing is applicable for sol-gel-derived fabrication of oxide films due to both fast metal-oxide formation by changing oxygen stoichiometry and chemical redistribution among different metals. Such detailed microscopic and chemical information will enhance our understanding of the resulting electrical properties of such relevant TSO films. 


\section{Abbreviations}

TSO: Transparent semiconducting oxide; ZTO: Zinc-tin-oxide; UV: Ultraviolet; TCO: Transparent conducting oxides; ZnO: Zinc oxide; XPS: X-ray photoemission spectroscopy; RT: Room temperature; TEM: Transmission electron microscopy; EELS: Electron energy loss spectroscopy; EDS: Energy dispersive $\mathrm{X}$-ray spectroscopy; $\mathrm{E}_{\mathrm{F}}$ : Fermi level; STEM: Scanning transmission electron microscopy

\section{Acknowledgements}

No applicable.

\section{Authors' contributions}

$J H K, J H P$, and YJC coordinated the initial idea of this study. SJ, HJK, BSJ, MH, and YJC carried out XPS analysis. KNY and EC analyzed optical spectroscopic data. JHK and YJC analyzed TEM experiments and drafted the manuscript. All authors have read, corrected, and approved the final manuscript.

\section{Funding}

This research was supported by Development of Fundamental Technology for Industrial Metrology funded by Korea Research Institute of Standards and Science (KRISS-2019-GP2019-0011). This work was supported by NRF2017R1C1B2004927 for H.J.K., NRF-2018R1A6A1A06024977 for B.S.J., NRF2015R1D1A1A01060381 for M.H., NRF-2017R1A2B4007782 for K.N.Y., and E.C. This research was financially supported by the Ministry of Trade, Industry and Energy (MOTIE) and Korea Institute for Advancement of Technology (KIAT) through the International Cooperative R\&D program (grant no. N0001819) and Basic Science Research Program through the National Research Foundation of Korea (NRF) funded by the Ministry of Science, ICT \& Future Planning (NRF-2017K1A3A1A19070288) for J.H.P. This work was supported by the 2017 Research Fund of the University of Seoul for Y.J.C. Experiments at PLS-II were supported in part by MSIP and POSTECH.

\section{Availability of data and materials}

Data sharing is not applicable to this article as no datasets were generated or analyzed during the current study.

\section{Competing interests}

The authors declare that they have no competing interests.

\section{Author details}

${ }^{1}$ Korea Research Institute of Standards and Science, 267 Gajeong-ro, Yuseong-gu, Daejeon 34113, Republic of Korea. ${ }^{2}$ Chemical Platform Technology Division, Korea Research Institute of Chemical Technology (KRICT), 141 Gajeong-Ro, Yuseong-Gu, Daejeon 34114, Republic of Korea. ${ }^{3}$ Department of Physics, University of Seoul, Seoul 02504, Republic of Korea. ${ }^{4} \mathrm{IT}$ Convergence and Components and Material Research Laboratory, Electronics and Telecommunications Research Institute, Daejeon 34129, Republic of Korea.

\section{Received: 14 October 2019 Accepted: 1 May 2020}

\section{Published online: 15 May 2020}

\section{References}

Bae S, Kim H, Lee Y, Xu X, Park J-S, Zheng Y, Balakrishnan J, Lei T, Ri Kim H, Song Yl, Kim Y-J, Kim KS, Özyilmaz B, Ahn J-H, Hong BH, lijima S. Roll-to-roll production of 30-inch graphene films for transparent electrodes Nat. Nanotechnol. 2010;5:574-8.

Banerjee AN, Ghosh CK, Chattopadhyay KK, Minoura H, Sarkar AK, Akiba A, Kamiya A, Endo T. Low-temperature deposition of $\mathrm{ZnO}$ thin films on PET and glass substrates by DC-sputtering technique. Thin Solid Films. 2006;496:112-6.

Chang YJ. Angle-resolved photoemission spectrscopy for chalcogenide and oxide heterostructures. Vacuum Magazine. 2018;5:10-7.

Charvin P, Abanades S, Lemont F, Flamant G. Experimental study of $\mathrm{SnO}_{2} / \mathrm{SnO} / \mathrm{Sn}$ thermochemical systems for solar production of hydrogen. AlChE J. 2008;54:2759-67.

Choi JS, Chang YJ, Woo S, Son Y-W, Park Y, Lee MJ, Byun I-S, Kim J-S, Choi C-G, Bostwick A, Rotenberg E, Park BH. Correlation between micrometer-scale ripple alignment and atomic-scale crystallographic orientation of monolayer graphene Sci. Rep. 2015:4:7263.

Fernandes C, Santa A, Santos Â, Bahubalindruni P, Deuermeier J, Martins R, Fortunato $E$, Barquinha P. A sustainable approach to flexible electronics with zinc-tin oxide thin-film transistors Adv. Electron. Mater. 2018;4:1800032.
Grigoropoulos C P, Bennett T D, Ho J-R, Xu X and Zhang X 1996 Heat and mass transfer in pulsed-laser-induced phase transformations Advances in Heat Transfer vol 28 (Elsevier) pp 75-144.

Hasan M, Oh J Y, Park J, Lim S C, Kim H O and Kang S Y 2012 Improved thin film transistor performance of solution-processed-zinc-oxide nanorods with spinon-glass capping layer Current Applied Physics 12 e18-e23.

Hong S-J, Song S-H, Kim BJ, Lee J-Y, Kim Y-S. ITO nanoparticles reused from ITO scraps and their applications to sputtering target for transparent conductive electrode layer. Nano Converg. 2017:4:23.

Izaki M, Shinagawa T, Mizuno K-T, Ida Y, Inaba M, Tasaka A. Electrochemically constructed $\mathrm{p}-\mathrm{Cu}, \mathrm{O} / \mathrm{n}-\mathrm{ZnO}$ heterojunction diode for photovoltaic device. J. Phys. Appl. Phys. 2007:40:3326-9.

Kang J, Dang V, Li H, Moon S, Li P, Kim Y, Kim C, Choi H, Liu Z, Lee H. InGaN-based photoanode with ZnO nanowires for water splitting. Nano Converg. 2016;3:34.

Kim HJ, Maeng M-J, Park JH, Kang MG, Kang CY, Park Y, Chang YJ. Chemical and structural analysis of low-temperature excimer-laser annealing in indium-tin oxide sol-gel films Curr. Appl. Phys. 2019;19:168-73.

Kim Y-H, Heo J-S, Kim T-H, Park S, Yoon M-H, Kim J, Oh MS, Yi G-R, Noh Y-Y, Park SK. Flexible metal-oxide devices made by room-temperature photochemical activation of sol-gel films. Nature. 2012;489:128-32.

Kwoka M, Ottaviano L, Passacantando M, Santucci S, Czempik G, Szuber J. XPS study of the surface chemistry of L-CVD SnO2 thin films after oxidation. Thin Solid Films. 2005:490:36-42.

Lee $\mathrm{H}$, Cho SW, Yi Y. Interfacial electronic structure for high performance organic devices. Current Applied Physics. 2016b;16:1533-49.

Lee $\mathrm{H}$, et al. Excimer laser annealing effects on AlGaN/GaN heterostructures. Current Applied Physics. 2016a;16:628-32.

Lee J-H, Kim J-Y, Kim J-H, Mirzaei A, Kim HW, Kim SS. Synthesis and gas sensing properties of membrane template-grown hollow ZnO nanowires. Nano Converg. 2017:4:27.

Lim LY, Lany S, Chang YJ, Rotenberg E, Zunger A, Toney MF. Angle-resolved photoemission and quasiparticle calculation of $\mathrm{ZnO}$ : the need for $\mathrm{d}$ band shift in oxide semiconductors. Phys. Rev. B. 2012;86:235113.

Lutz P, Moser S, Jovic V, Chang YJ, Koch RJ, Ulstrup S, Oh JS, Moreschini L, Fatale $\mathrm{S}$, Grioni M, Jozwiak C. Volatile two-dimensional electron gas in ultrathin BaTiO 3 films. Physical Review Materials. 2018;2:094411.

Minami T. Substitution of transparent conducting oxide thin films for indium tin oxide transparent electrode applications. Thin Solid Films. 2008;516:1314-21.

Mishra KC, Johnson KH, Schmidt PC. Electronic structure of antimony-doped tin oxide Phys. Rev. B. 1995;51:13972-6.

Nakajima T, Tsuchiya T, Kumagai T. Perfect uniaxial growth of Dion- Jacobson Perovskite RbLaNb2O7 thin films under pulsed photothermal gradient heating. Crystal growth \& design. 2010;10:4861-7.

Noh M, Seo I, Park J, Chung J-S, Lee YS, Kim HJ, Chang YJ, Park J-H, Kang MG, Kang CY. Spectroscopic ellipsometry investigation on the excimer laser annealed indium thin oxide sol-gel films Curr. Appl. Phys. 2016;16:145-9.

Oh SH, Ferblantier G, Park YS, Schmerber G, Dinia A, Slaoui A, Jo W. Lowtemperature growth and electronic structures of ambipolar Yb-doped zinc tin oxide transparent thin films Appl. Surf. Sci. 2018:441:49-54.

Pennycook S J 2002 Structure determination through Z-contrast microscopy Advances in imaging and electron physics vol 123 (Elsevier) pp 173-206.

Pennycook SJ, Li C, Li M, Tang C, Okunishi E, Varela M, Kim Y-M, Jang JH. Material structure, properties, and dynamics through scanning transmission electron microscopy. J. Anal. Sci. Technol. 2018;9:11.

Sandu CS, et al. Densification and crystallization of SnO2: Sb sol-gel films using excimer laser annealing. Applied Surface Science. 2003:208:382-7.

Seiler H, Haas U, Körtje K-H, Ocker B. Investigation of metal cluster layers by EELS Microsc. Microanal. Microstruct. 1991;2:191-201.

Seo D, Yu K, Jun Chang Y, Sohn E, Hoon Kim K and Choi E J 2014 Infrared-optical spectroscopy of transparent conducting perovskite $(\mathrm{La}, \mathrm{Ba}) \mathrm{SnO}_{3}$ thin films Appl. Phys. Lett. 104022102.

Wencka M, Kovač J, Dasireddy VDBC, Likozar B, Jelen A, Vrtnik S, Gille P, Kim HJ, Dolinšek J. The effect of surface oxidation on the catalytic properties of Ga3Ni2 intermetallic compound for carbon dioxide reduction. J. Anal. Sci. Technol. 2018;9:12

Widder K, Knupfer M, Knauff O, Fink J. Plasmon behavior of Zn from electronenergy-loss spectroscopy Phys. Rev. B. 1997:56:10154-60.

\section{Publisher's Note}

Springer Nature remains neutral with regard to jurisdictional claims in published maps and institutional affiliations. 